\title{
La Embarazada Añosa
}

Guillermo López Escobar, M. D.

\section{AGRADECIMIENTOS}

Los autores desean expresar sus agradecimientos a todas las entidades $e$ individuos que en una $u$ otra forma han contribuido en la elaboración y preparación de este documento y particularmente a los investigadores de la red hospitalaria del Programa Regional de Investigaciones en Fecundidad (PRIF), y a los del Ministerio de Salud.

\section{LA EMBARAZADA AÑOSA}

La embarazada por encima de los 35 años ha constituido siempre motivo de preocupación, tanto en el sector médico como a nivel familiar. Dentro de la clasificación del riesgo obstétrico, este embarazo ha sido catalogado como de alto riesgo. Su cuantificación en cifras colombianas, y su estudio, parece importante.

\section{MATERIAL Y METODOS}

En la investigación sobre el parto hospitalario en Colombia, Ilevada a cabo a través de una muestra nacional de 40 hospitales, por medio de un cuestionario semiprospectivo precodificado (IFRP-PRIF), sobre un total de 13.450 casos recolectados de marzo a octubre de 1977, se encontraron 1.178 casos de embarazadas de más de 35 años, las cuales representan el $8.7 \%$.

El presente trabajo analiza 1.168 de estos casos de embarazadas añosas
Germán Riaño Gamboa, M. D.

(se han descartado 10 casos, por razones de defecto de recolección), de los cuales $884(6.6 \%)$ corresponden a mujeres de $35-39$ años y $284(2.1 \%)$ a mujeres de 40 a 48 años. Además, se compara este grupo con 11.040 casos de partos en mujeres de 18 a 34 años, correspondiente al mismo estudio.

\section{RESULTADOS}

\section{Paridad}

Como es de esperar, la mayoría de estas mujeres eran grandes multíparas, de 4 o más partos, $(71.3 \%)$. Sin embargo, había un $6 \%$ de primíparas (Cuadro No. 1).

\section{Duración estimada del embarazo}

La duración del embarazo en semanas fue similar en los grupos. Hubo un $1 \%$ de embarazos de 43 semanas o más y $94.1 \%$ de 36 a 42 semanas en el grupo de 35 a 48 años, similar, $95.3 \%$ en el grupo control de 18 a 34 , lo cual los hace comparables.

\section{Principal patología perinatal}

La patología perinatal inmediata es más abundante en la embarazada añosa (Cuadro No. 2), particularmente en lo que hace referencia a la enfermedad hipertensiva, transtornos cardiovasculares, incluyendo tromboflebitis; es ligeramente mayor el sangrado sobre todo en las mujeres de 40-48 años. 
CUADRO No. 1

EDAD SEGUN PARIDAD

\begin{tabular}{|c|c|c|c|c|c|}
\hline \multirow[t]{2}{*}{ PARIDAD } & & \multicolumn{2}{|r|}{$E$} & A & \\
\hline & & $35-39$ & $40-48$ & $35-48$ & $\begin{array}{c}\text { Grupo C/parativo } \\
18 \cdot 34\end{array}$ \\
\hline Primíparas & $\begin{array}{r}\mathrm{N} \\
\%\end{array}$ & $\begin{array}{c}(64) \\
7.2\end{array}$ & $\begin{array}{l}(6) \\
2.1\end{array}$ & 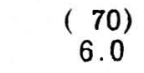 & $\begin{array}{c}(4.399) \\
39.9\end{array}$ \\
\hline Multíparas 1-3 & $\underset{\%}{N}$ & $\begin{array}{l}(217) \\
24.5\end{array}$ & $\begin{array}{l}(48) \\
16.6\end{array}$ & $\begin{array}{l}(265) \\
22.7\end{array}$ & $\begin{array}{c}(5.372) \\
48.7\end{array}$ \\
\hline $\begin{array}{l}\text { Gran-Multíparas } \\
4 \text { o más }\end{array}$ & $\underset{\%}{\mathrm{~N}}$ & $\begin{array}{l}(603) \\
68.3\end{array}$ & $\begin{array}{l}(230) \\
81.3\end{array}$ & $\begin{array}{l}(833) \\
71.3\end{array}$ & $\begin{array}{c}(1.269) \\
11.5\end{array}$ \\
\hline
\end{tabular}

FUENTE: G. López. "La E. Añosa". CCrp-prif 901.

\section{Tipo de trabajo de parto}

La mayoría de los trabajos de parto fueron espontáneos. En el grupo de 3539 hubo un $4.6 \%$ de partos inducidos y en los de $40-48$ hubo $6.7 \%$. En el grupo comparativo de 18 a 34 los inducidos fueron $4.1 \%$

\section{Tipo de presentación}

En el grupo de 35-48 años, el $75.9 \%$ de los casos fueron presentaciones de vértice occipito anteriores y $17 \%$ occipito transversales y occipito posteriores, cuando en el grupo comparativo de 18-34 hubo $80.5 \%$ anteriores y $14.0 \%$ OT y OP. La mayor diferencia está en las presentaciones de hombro, que constituyen el $1.2 \%$ en las mujeres de edad y en el otro sólo $0.4 \%$ (Cuadro No. 3). Esto muy probablemente se relaciona con la multiparidad, mucho mayor en el grupo de mayor edad. Así también, los 6 casos de presentación de cara o frente $(0.5 \%)$ fueron todos en grandes multíparas.

CUADRO No. 2

PRINCIPAL PATOLOGIA PRENATAL

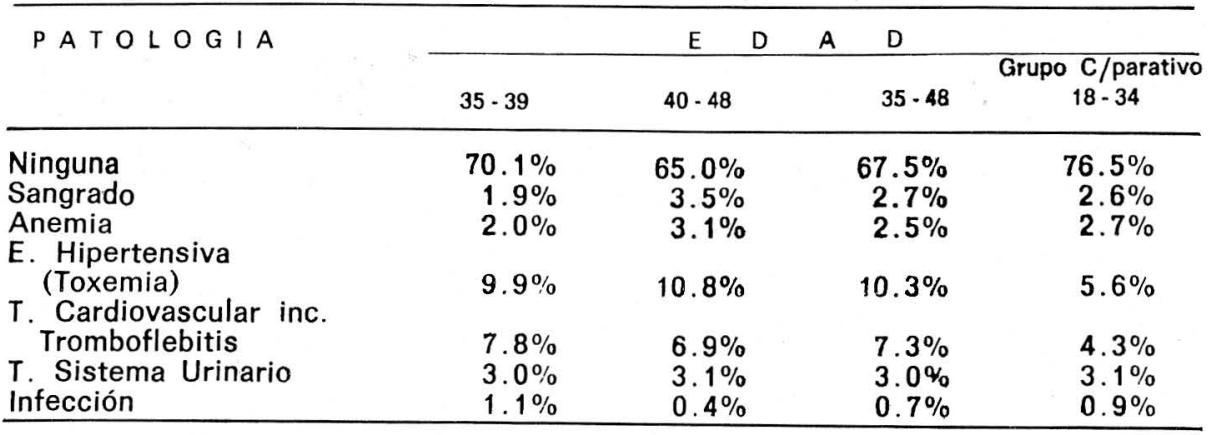

FUENTE: G. López. "La E. Añosa". CCRP.PRIF 901. 
CUADRO No. 3

TIPO DE PRESENTACION

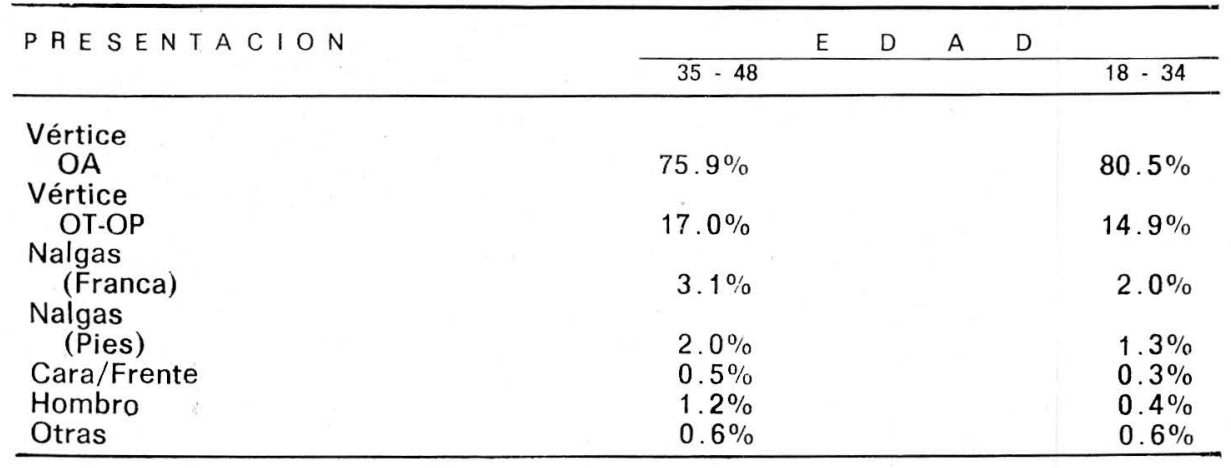

FUENTE: G. López, "La E. Añosa".

\section{Anestesia}

Un buen número $(65.5 \%)$ de las mujeres de edad no recibieron anestesia 0 solo psicoprofilaxis para su parto. Llama la atención que la anestesia local se utilizó en solo $9.3 \%(N=108)$ de los casos, comparativamente con el grupo de 18 a 34 años que la empleó en $35.1 \%$ $(\mathrm{N}=-4.683)$. La anestesia espinal o epidural se usó en el $11.4 \%(N=133)$. (Cuadro No. 4).

\section{Episiotomía}

A un buen porcentaje, $89.8 \%(\mathrm{~N}=$ 1.049) de las pacientes de edad, no se les practicó episiotomía. Esto es aún más llamativo al encontrar que en el grupo de primíparas en más de la mitad, $55.7 \%$, no hubo episiotomía, que se supone es un procedimiento que ayuda no sólo a salvaguardiar el traumatismo del periné materno, sino del feto al nacer. Tanto más, cuanto que en

CUADRO No. 4

A N E S T E S I A

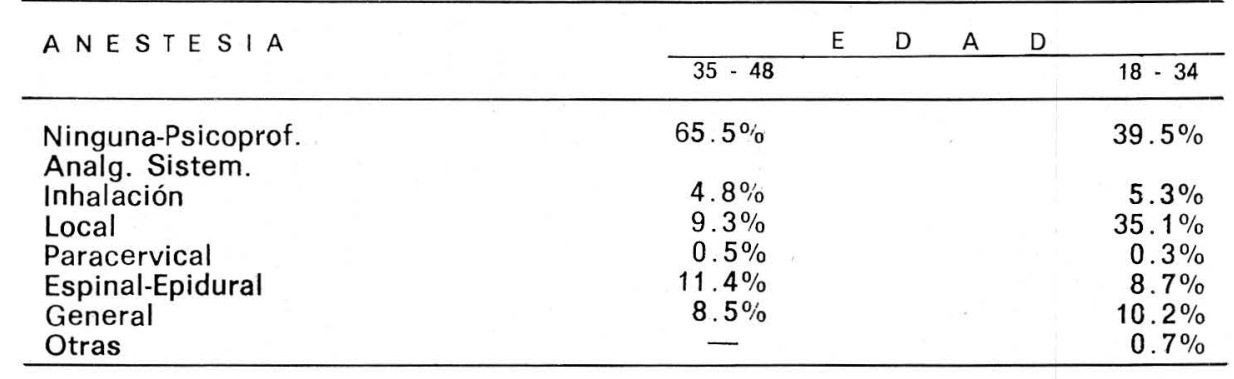

FUENTE: G. López, "La E. Añosa" CCRP-PRIF 901. 
CUADRO NO. 5

E PISIOT O M I A

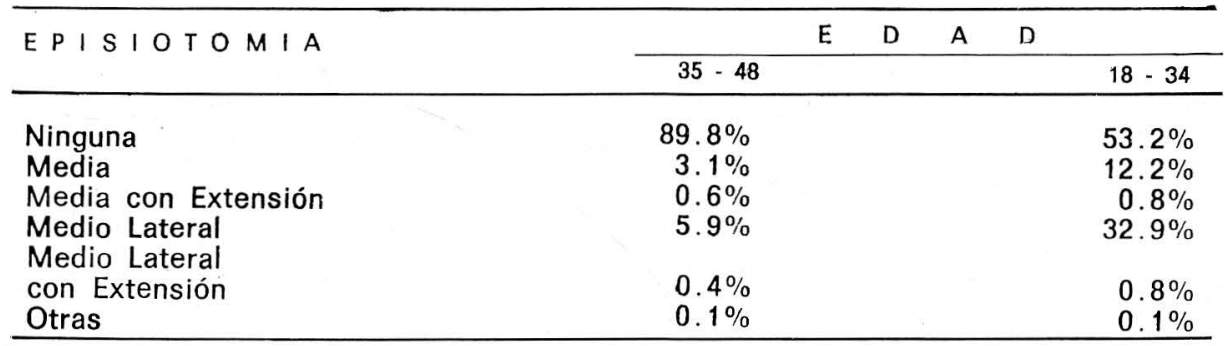

FUENTE: G. López, "La E. Añosa" CCRP.PRIF 901.

el grupo comparativo de 18 a 34 años, el porcentaje de no episiotomías es más bajo, $53.0 \%$ para todas, y $19.0 \%$ para las primíparas. Agréguese a esto que el único caso de lesión en la vejiga no tuvo episiotomía, ni tampoco hubo episiotomía en $75 \%$ de los casos de lesión en el periné y en $77 \%$ de los de lesión en la vulva. (Cuadro No. 5).

\section{Tipo de parto}

La gran mayoría de las mujeres de 35-48 años tuvo el parto espontánea- mente, $82.4 \%$. Es sin embargo importante mencionar que el porcentaje de cesáreas es el doble, $13.2 \%(\mathrm{~N}=154)$, que en el grupo comparativo de 18-34 años, $6.6 \%(N=729)$. Como es de esperar en las primíparas de edad casi la mitad, $41.4 \%(\mathrm{~N}=29)$ terminaron en cesárea, cuando en el grupo comparativo las primíparas, en sólo $8.1 \%(\mathrm{~N}=359)$ la tuvieron. La otra mitad tuvo el parto espontáneamente, excepto $0.9 \%$ con extractor al vacio y $2.1 \%$ con fórceps bajo. (Cuadro No. 6).

CUADRO No. 6

TIPO DE PARTO

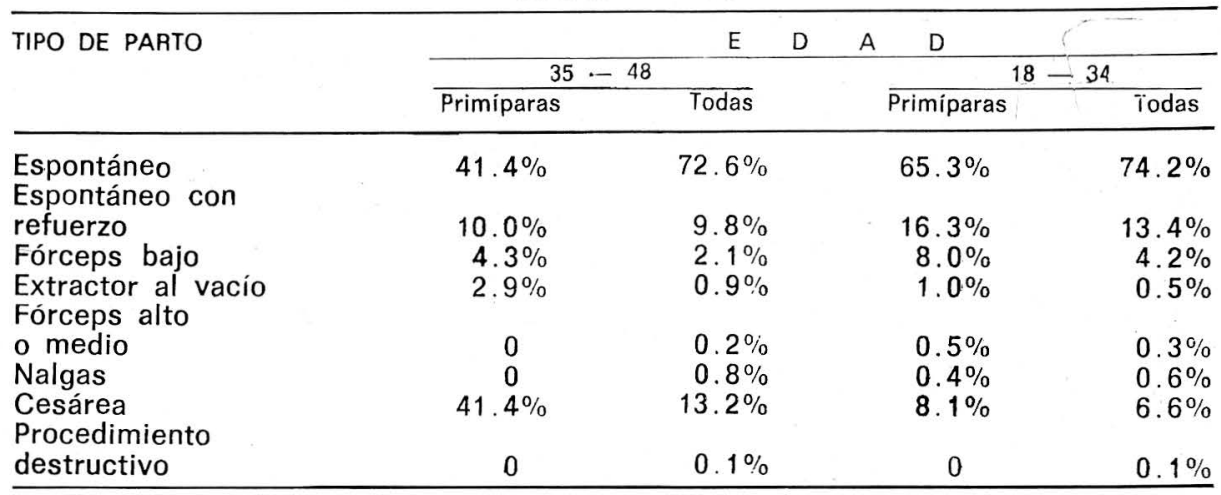

FUENTE: G. López, "La E. Añosa" CCRP-PRIF 901 


\section{Lesión principal durante el trabajo o parto}

Los traumatismos y lesiones del trabajo y el parto fueron escasos. El $91.9 \%$ $(N=-1.073)$ no tuvo ninguna lesión; un $4.8 \%(N=56)$ sufrió desgarros perineales y el $1.8 \%(N=21)$ lesiones de vagina. Sólo hubo un caso de lesión de la vejiga. La comparación con el grupo de 18-34 es favorable, pues éste tuvo sólo $85.0 \%(\mathrm{~N}=9.382)$ de no lesiones. La explicación probablemente estriba en que en el grupo de edad el porcentaje de cesáreas es mucho mayor, como ya se vio anteriormente, de modo que la posibilidad de sufrir lesiones perineales o traumatismos locales es entonces menor.

\section{Complicación primaria del trabajo o parto}

Un poco más de las cuatro quintas partes de los casos no tuvieron ninguna complicación, $84.3 \%(\mathrm{~N}=985)$. Esta cifra baja a $80.3 \%$ si se toman únicamente los casos de 40 o más años y es mayor, $90.2 \%$, en el grupo comparativo de 18 a 34 años. De resto, es necesario destacar $5.9 \%(N=69)$ de casos de trabajo prolongado; $2.3 \%$ $(\mathrm{N}=-27)$ de placenta previa; $2.3 \%$ de hipotonía uterina y $1.2 \%$ de abruptio. En el grupo comparativo de 18 a 34 años, las cifras son ligeramente más bajas. Sorprendentemente el abruptio fue menor en $1 \%$, en las mujeres de 40 a 48 años frente a las de 35-39 años. (Cuadro No. 7)

\section{Complicación secundaria del trabajo o parto}

Las complicaciones secundarias más importantes fueron la hemorragia postparto, que se sucedió en $2.6 \%(\mathrm{~N}=$ 30) de los casos de mujeres de edad, y el trabajo prolongado en $1.5 \%$; respecto a este último la diferencia es notoria entre el grupo de 35-39, que tuvo $0.9 \%(\mathrm{~N}=8)$ de trabajo prolongado. con el de 40-48 años que lo hizo en $3.2 \%(\mathrm{~N}=9)$; el grupo comparativo de 18-34 años muestra cifras similares $0.9 \%(N=100)$ de trabajo prolongado y menor, $0.8 \% \quad(N=-85)$, de hemorragia post-parto.

\section{Duración del trabajo de parto}

La duración no parece estar aumentada en comparación con el grupo de 18-34 años; sin embargo, el porcentaje de primíparas y grandes multíparas diferentes en los diversos grupos hace la comparación global difícil; por esto parece más juicioso comparar según paridad aisladamente. Un $25.7 \%(\mathrm{~N}=18)$ de las primíparas de $35-48$ años tuvieron 13 o más horas de trabajo de parto, en comparación con $32.3 \%(N=1.419)$

CUADRO No. 7

COMPLICACION PRIMARIA DEL TRABAJO O PARTO

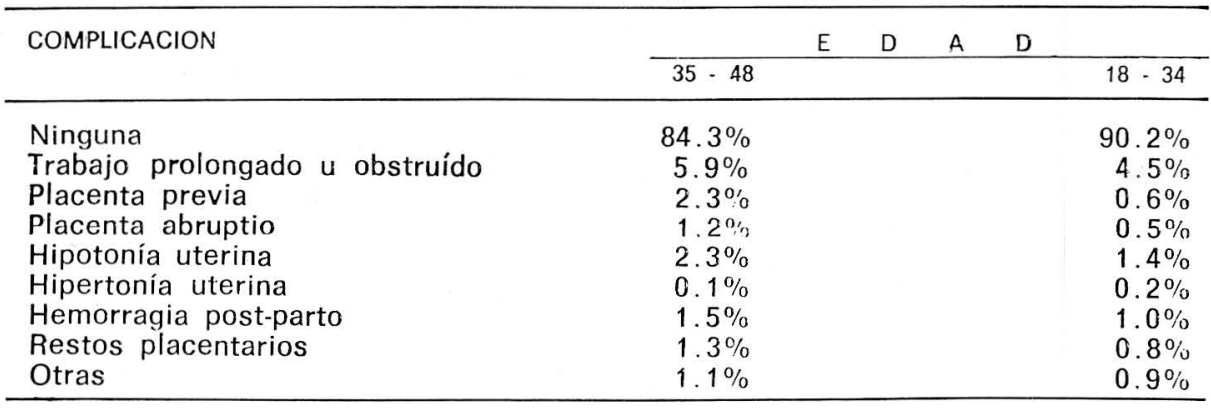

FUENTE: G. López, "La E. Añosa", CCRP.PRIF 901 
del grupo de 18-34 años. En relación con las grandes multíparas de 4 y más partos, un $15.1 \%(\mathrm{~N}=126)$ de las de 35-48 años tuvieron 13 o más horas de trabajo, en comparación con $14.4 \%$ $(N=182)$ de las del grupo de 18-34. Este hallazgo de duración similar o menor del trabajo en las primíparas añosas muy probablemente sea debido ai alto porcentaje, casi la mitad de cesáreas en estas primíparas, lo cual obviamente produce un sesgo.(CuadroNo.8).

\section{Atención del parto}

El estudio de la atención del parto, según el tipo de profesional que la atendió, revela que en este grupo de edad, las cesáreas las realizó el especialista en $74 \%$ de los casos, el médico general en el $25.3 \%$ y estudiantes de medicina en $0.6 \%$. Las nalgas en $55.6 \%$ el especialista, $11.1 \%$ el médico general y $33.3 \%$ estudiantes de medicina. Las cifras son globalmente para cada profesional similares en el grupo de 18-34 años; esto significaría que la atención de la embarazada añosa se llevó a cabo sin darle una prerrogativa especial.

\section{Peso de neonato}

En el grupo de 35 a 39 años el $10.4 \%$ de los recién nacidos pesó menos de
2.500 gms; en cambio en el grupo de 40-48 este porcentaje fue mayor, $14.5 \%$. En conjunto el grupo de las añosas de 35 a 48 años tuvo también un porcentaje mayor, $11.4 \%$ de menos de 2.500 gms, que en el grupo de 18-34, que fue de $8.3 \%$. Así, también fue mayor el porcentaje de los de más de $4.000 \mathrm{gms}$ : $10.6 \%$ para las de $35-48$ y $5.2 \%$ para las de 18-34.

\section{Apgar al minuto}

En este grupo de edad, $80.5 \%$ de los nacidos tenían un apgar de 8 o más al minuto y solo $7.4 \%$ con apgar 0 . Las cifras correspondientes para el grupo comparativo de $18-34$ son $82.4 \%$ y

\section{$3.7 \%$ respectivamente}

\section{Estado del neonato}

La comparación entre el grupo de 35 a 39 con el de 40 a 48, muestra cómo el riesgo fetal aumenta definitivamente con la edad materna: $86.7 \%(\mathrm{~N}=766)$ de los casos del primer grupo eran normales al nacer, cuando $83.3 \%$ en el segundo $(\mathrm{N}=238)$; en relación con el grupo de 18-34 esta cifra es de $91.4 \%$ normales $(\mathrm{N}=10.089)$. Aún más importante es la diferencia en el porcentaje de malformaciones: $2.2 \%$ en las de 40 a $48,0.8 \%$ en las de 35 a 39 y $0.6 \%$ en las de $18-34$. Es interesant3

CUADRO No. 8

DURACION DEL TRABAJO

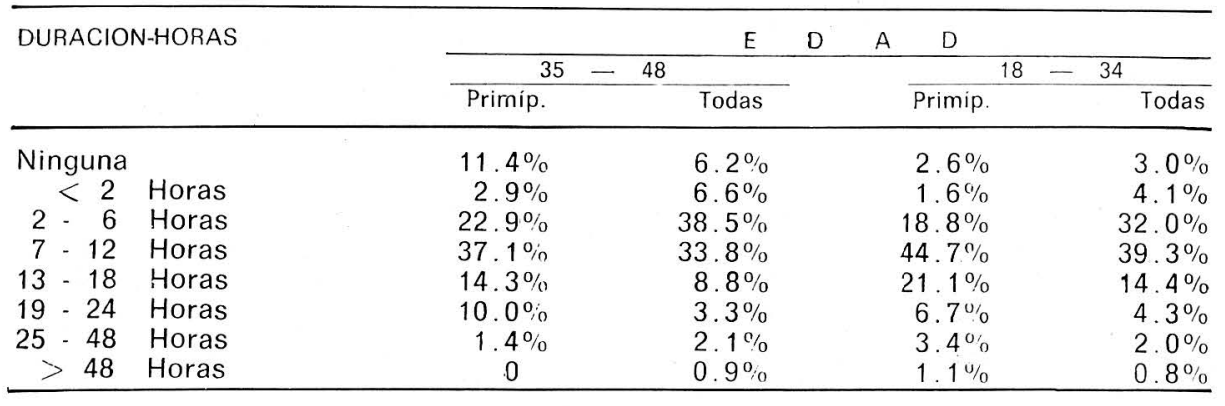

FUENTE: G. López, "La E. Añosa", CCRP-PRIF 901. 
CUADRO NO. 9

ESTADO DEL NEONATO

\begin{tabular}{lrrr}
\hline E S T A D O & \multicolumn{3}{c}{ E D } \\
\cline { 2 - 4 } & $35-39$ & $40-48$ & $13-34$ \\
\hline & & & G. C/parativc \\
Normal & $86.7 \%$ & $83.8 \%$ & $91.4 \%$ \\
Sufrimiento intra-parto & $4.4 \%$ & $5.3 \%$ & $3.4 \%$ \\
Malformación menor & $0.1 \%$ & $1.1 \%$ & $0.4 \%$ \\
Malformación mayor & $0.7 \%$ & $1.1 \%$ & $0.2 \%$ \\
Sindr. depresión, respiratoria & $2.6 \%$ & $2.8 \%$ & $1.8 \%$ \\
Ictericia & $0.5 \%$ & $0.4 \%$ & $0.2 \%$ \\
Sepsis neonatorum & $0.3 \%$ & $0.7 \%$ & $0.2 \%$ \\
Trauma & $0.1 \%$ & $0.4 \%$ & $0.1 \%$ \\
Otro & $4.6 \%$ & $4.6 \%$ & $2.4 \%$ \\
\hline
\end{tabular}

FUENTE: G. López, "La E. Añosa", CCRP.PRIF 901.

anotar que en las añosas todas las malformaciones se sucedieron en multíparas. Las otras cifras, ligeramente mayores en las de mayor edad, corresponden a sufrimiento intraparto, 4 a $5 \%$ y depresión respiratoria al nacer, cerca de $3 \%$. (Cuadro 9).

\section{Estado puerperal de la madre}

Aquí también la diferencia es aún más notoria cuanto mayor edad tenga el grupo. El porcentaje de madres normales en el puerperio es solo de $90.1 \%$ en las que tenían de 40-48 años; de $92.5 \%$ en las de $35-39$ y 96.0 en las de 18-34 años. El sangrado y la tiebre que requirieron tratamiento fueron más frecuentes en las añosas. Pero sobre todo, la mortalidad materna es más alta. Aunque el estudio metodológicamente, por el tiempo en que se hizo la recolección, no es completamente adecuado para estudiar mortalidad materna en sí, la comparación entre los grupos de edades parece sin embargo valedera, pues las condiciones son similares. Así, las cifras de mortalidad materna fueron: 20 por 10.000 en el grupo de 18-34 años y 30 por 10.000 en el total de las de 35-48 años; esta cifra se hace aún más notoria si se toma la mortalidad materna únicamente para el

CUADRO No. 10

ESTADO PUERPERAL MATERNO

\begin{tabular}{lrrr}
\hline E S T A D O & \multicolumn{3}{c}{ E } \\
\cline { 2 - 4 } & $35-39$ & $40-48$ & $18-34$ \\
\hline & & & G. C/parativo \\
Normal & $92.5 \%$ & $90.1 \%$ & $96.0 \%$ \\
Fiebre que requirió tratamiento & $3.8 \%$ & $3.5 \%$ & $1.7 \%$ \\
Sangrado que requirió tratamiento & $2.4 \%$ & $4.2 \%$ & $1.3 \%$ \\
Infección tracto urinario & $0.1 \%$ & $0.4 \%$ & $0.1 \%$ \\
Mastitis & $0.1 \%$ & $0.4 \%$ & $0.0 \%$ \\
Muerte & $0.2 \%$ & $0.7 \%$ & $0.2 \%$ \\
Otros & $0.8 \%$ & $0.7 \%$ & $0.7 \%$ \\
\hline
\end{tabular}

FUENTE: G. López, "La E. Añosa", CCRP-PRIF 901. 
grupo de 40 a 48 que es de 70 por 10.000 , cifra comparable a la que se ha citado en relación con el aborto inducido (1). (Cuadro No. 10).

\section{Noches de hospitalización}

La permanencia en el hospital después del parto aumenta en un buen porcentaje de los casos de mayor edad. Asi, $14.2 \%$ del grupo de $35-39$ años permaneció 4 o más noches hospitalizada post-partum, $19.5 \%$ en los de 40 a 48 años. En el grupo comparativo de 18-34 años, la cifra es prácticamente la mitad, $8.2 \%$ con 4 o más noches de hospitalización.

\section{MORTALIDAD PERINATAL}

La mortalidad perinatal inmediatamente antes del parto, intra-parto y post-parto, mientras dura la hospitalización de la madre, es mayor en el grupo de las añosas, $6.5 \%$, en comparación con el grupo de 18-34 años en donde sólo es de $2.6 \%$, prácticamente la tercera parte. No obstante, esta mortalidad merece analizarse a través de diversos parámetros que permitan una más juiciosa comparación. Los siguientes son algunos de estos cruces:

\section{Peso y mortalidad perinatal}

Como se vio anteriormente en las mujeres de edad, 35 a 48 años, los recién nacidos de menos de $2.500 \mathrm{gms}$. tienen mayor porcentaje que las de 18 a 34. Pero es más, comparando entre sí los grupos del mismo peso, la mortalidad perinatal es siempre mayor en las añosas. Así, en las de 35-48 años con neonatos de menos de 2.500 gms., hubo $30 \%$ de mortalidad perinatal frente a $13 \%$ en las de 18 a 34 años, también con neonatos de menos de 2.500 gms. En el grupo de 35-48 años con neonatos de 2.500 gms. a 4.499 , hubo $3.3 \%$ de muertes perinatales en comparación con $1.54 \%$ para las de 18-34; en el grupo con más de $4.500 \mathrm{gms}$. en las de 35 a 48 , hubo $5.8 \%$ de mortalidad perinatal frente a $1.2 \%$ de las de 20 a 34 años. (Cuadro No. 11).

\section{Complicación primaria del trabajo-parto y mortalidad perinatal}

Anteriormente se describió cómo las complicaciones primarias son mayores en el grupo de añosas. Pero, además, aquí también la mortalidad perinatal es mayor en ellas comparando las diversas edades dentro de una misma complicación. Así, el grupo de 35-48 años sin ninguna complicación tuvo $4.3 \%$ de mortalidad perinatal, cuando el de 18 a 34 sin ninguna complicación, solo tuvo $1.7 \%$. Los 69 casos de trabajo obstruído o prolongado en las de 35-48 tuvieron $13.0 \%$ de muertes perinatales, cuando los 500 casos de 18-34 sólo $8.2 \%$. En las placentas previas, los 27 casos del grupo de 35-48 años tuvieron $33.3 \%$ de muertes perinatales cuando el grupo de 18-34 tuvo sólo $20.9 \%$. La hipotonía uterina en las de 35-48 tuvo $14.8 \%$ de muertes perinatales frente a $5.1 \%$ en las de 18-34. La placenta

CUADRO NO. 11

PESO Y MORTALIDAD PERINATAL

\begin{tabular}{rlrc}
\hline$P E S O$ & \multicolumn{2}{c}{ MORTALIDAD PERINATAL } \\
\cline { 3 - 3 } & & & $18 \cdot 34$ \\
\hline & $-2499 \mathrm{gms}$ & $30.0 \%$ & G. C/parativo \\
$2500-4499 \mathrm{gms}$ & $3.3 \%$ & $15 \%$ \\
$4500-$ y más & $5.8 \%$ & $1.5 \%$ \\
\hline
\end{tabular}

FUENTE: G. López, "La E. Añosa", CCRP.PRIF 901. 
abruptio fue igualmente letal para el feto en todas las edades: $50 \%$ en las de $35-48$ y $52.6 \%$ en las de $18-34$ años.

En general, la mortalidad ante o intra-parto es 2-3 veces mayor que la post-parto, lo cual es lógico si se piensa que las muertes neonatales registradas solo corresponden al tiempo de estadía hospitalaria de la madre. (Cuadro No. 12).

\section{Ruptura de las membranas y mortalidad perinatal}

Las cifras presentadas en el Cuadro No. 13, corroboran la experiencia obstétrica en el sentido que la ruptura prematura de las membranas 24 horas o más, antes del parto, entraña un mayor peligro para el feto; casi una cuarta parte, $23.3 \%$ de los casos en que esto sucedió, terminaron en feto muerto intra o post-parto.

Aún la ruptura antes de los $4 \mathrm{cms}$. acarrea un porcentaje ligeramente mayor de mortalidad, mucho más si es artificial $(15 \%)$ en comparación con $4.6 \%$ en las que hubo ruptura artificial después de los $4 \mathrm{cms}$. Pero lo más significativo es que comparativamente a nivel de cada categoría, la mortalidad perinatal es mayor en el grupo de mayor edad. indicando siempre el mayor riesgo pa- ra este grupo, aún aquí con un parámetro que a primera vista parecería no tener una relación tan estrecha, (aunque el análisis se ha hecho diferenciando las muertes fetales post-parto, para efectos del cuadro, estas se han unido)

\section{Tipo del trabajo y muerte fetal}

El parto espontáneo en el grupo de 35-48 tuvo una mortalidad perinatal de $5.1 \%$. Todos los otros grupos tienen mayor mortalidad con excepción del grupo de parto inducido con ruptura artificial de las membranas (RAM) que tuvo $0 \%$ de muertes perinatales y el grupo de parto espontáneo más RAM y ocitócicos que tuvo $2.5 \%$. Aquí de nuevo la comparación con el grupo de 18 a 34 revela que no importa en cuál categoria la mortalidad perinatal de la añosa, es siempre mayor. Así, en el grupo de 35-48 años el trabajo de parto espontáneo tiene $5.1 \%$ de mortalidad, cuando en el grupo comparativo de 18 a 34 años tiene $2.6 \%$ y, a excepción de las dos categorias mencionadas, la mortalidad perinatal es siempre un poco mayor en las añosas.

\section{Tipo de presentación y muerte fetal}

La mortalidad perinatal sigue los patrones esperados. Es mayor en la pre-

CUADRO No. 12

\section{COMPLICACION PRIMARIA DEL TRABAJO - PARTO Y MORTALIDAD PERINATAL}

\begin{tabular}{lcc}
\hline COMPLICACION & \multicolumn{2}{c}{ MUERTES PERINATALES } \\
\cline { 2 - 3 } & $35 \cdot 48$ & $18-34$ \\
\hline & & G. C/parativo \\
Ninguna & $4.3 \%$ & $1.7 \%$ \\
Trabajo prolongado u obstruido & $13.0 \%$ & $8.2 \%$ \\
Placenta previa & $33.3 \%$ & $20.9 \%$ \\
Placenta abruptio & $50.0 \%$ & $52.6 \%$ \\
Utero hipotónico & $14.8 \%$ & $5.1 \%$ \\
Utero hipertónico & - & - \\
Hemorragia post-parto & - & $10.5 \%$ \\
Pestos placentarios & $6.6 \%$ & \\
\hline
\end{tabular}

FUENTE: G. López, "La E. Añosa". CCRP.PRif 901. 
CUADRO NO. 13

RUPTURA DE MEMBRANAS Y MORTALIDAD PERINATAL

\begin{tabular}{|c|c|c|c|c|}
\hline \multirow{3}{*}{$\begin{array}{l}\text { RUPTURA DE } \\
\text { MEMBRANAS }\end{array}$} & \multicolumn{4}{|c|}{ MORTALIDAD FETAL } \\
\hline & \multirow{2}{*}{$\begin{array}{r}35 \\
\text { Vivos }\end{array}$} & 48 & \multirow{2}{*}{$\begin{array}{l}18 \\
\text { Vivos }\end{array}$} & -34 \\
\hline & & $\begin{array}{l}\text { Ante-Intra } \\
\text { Post-Partum }\end{array}$ & & $\begin{array}{c}\text { Ante-Intra } \\
\text { Post-Partum }\end{array}$ \\
\hline $\begin{array}{l}\text { Prematura } 24 \text { horas o } \\
+ \text { antes del parto } \\
\text { Prematura }<\text { de } 24 \text { horas }\end{array}$ & $76.7 \%$ & $23.3 \%$ & $90.7 \%$ & $9.4 \%$ \\
\hline $\begin{array}{l}\text { antes del parto } \\
\text { Espontánea }<\text { de } 4\end{array}$ & $95.7 \%$ & $4.2 \%$ & $95.6 \%$ & $4.4 \%$ \\
\hline $\begin{array}{l}\text { cms. dilatación } \\
\text { Espontánea }>\text { de } 4\end{array}$ & $89.6^{\circ} \%$ & $10.5^{\%} \mathrm{o}$ & $98.4 \%$ & $1.6^{\circ} \%$ \\
\hline $\begin{array}{l}\text { cms dilatación } \\
\text { Espontánea dilatación }\end{array}$ & $95.8 \%$ & $4.1^{\circ} \circ$ & $98.4 \%$ & $1.6 \%$ \\
\hline $\begin{array}{l}\text { completa } \\
\text { Espontánea dilatacıon }\end{array}$ & $97.3^{\circ} 0$ & $2.7^{\circ} \mathrm{c}$ & $98.0 \%$ & $2.0^{\circ} \%$ \\
\hline $\begin{array}{l}\text { desconocida } \\
\text { Artificial }<4 \mathrm{cms} \text {. }\end{array}$ & $93.3 \%$ & $6.7^{\circ} \circ$ & $97.1 \%$ & $2.9 \%$ \\
\hline $\begin{array}{l}\text { dilatación } \\
\text { Artificial }>4 \mathrm{cms} \text {. }\end{array}$ & $85.0 \%$ & $15.0 \%$ & $89.0 \%$ & $11.0 \%$ \\
\hline dilatación & $95.4^{\circ} \circ$ & $4.6 \%$ & $98.4 \%$ & $1.6 \%$ \\
\hline Durante la cesárea & $84.4^{\circ}{ }_{i}$ & $15.7 \%$ & $92.5 \%$ & $7.4 \%$ \\
\hline
\end{tabular}

FUENTE: G. López, "La E. Añosa", CCRP-PRIF 901

sentación de nalgas que en la de vertice, mayor en las de cara y frente y aún mayor en la de hombro. La comparación con el grupo de 18-34 años una vez más revela el riesgo mayor en las mujeres de mayor edad. Para señalar sólo unos ejemplos: el vértice en OA acusa $5.7 \%$ de mortalidad perinatal en las de 35 a 48 años cuando en las de 18-34 sólo tiene 2.0. En las nalgas francas, las cifras son $19.5 \%$ y $12.9 \%$; en todas las presentaciones sigue el mismo patrón a excepción de las de hombro que tienen mortalidad similar. (Cuadro No. 14).

CUADRO No. 14

TIPO DE PRESENTACION Y MORTALIDAD PERINATAL

\begin{tabular}{lrr}
\hline PRESENTACION & \multicolumn{2}{c}{$\begin{array}{c}\text { ANTE-INTRA-POST-PARTUM } \\
\text { MUERTES }\end{array}$} \\
\cline { 2 - 3 } & 35.48 & $18-34$ \\
\hline Vértice OA & & G $/$ parativo \\
Vértice OT-OP & $5.7 \%$ & $2.0 \%$ \\
Nalgas franca & $4.0 \%$ & $2.1 \%$ \\
Nalgas-pies & $19.5 \%$ & $12.9 \%$ \\
Cara-frente & $17.3 \%$ & $11.4 \%$ \\
Hombro & $33.4 \%$ & $3.2 \%$ \\
\hline
\end{tabular}

FUENTE: G. López. "La E. Añosa”, CCRP.PRIF 901. 


\section{Anestesia y muerte fetal}

El parto sin anestesia o la psicoprofilaxis tiene una mortalidad perinatal de $5.8 \%$ en las de $35-48$ y de $3.3 \%$ en el grupo de 18-34. Las cifras para la anestesia local son $2.8 \%$ y $1.3 \%$ respectivamente y para la anestesia epidural o espinal son $9.8 \%$ y $2.9 \%$.

\section{Tipo de parto y mortalidad perinatal}

Finalmente, al considerar el tipo de parto los hallazgos muestran otra vez la mayor mortalidad perinatal de las embarazadas de edad. Así, además de la diferencia en el parto espontáneo que ya ha sido señalada, en el parto de nalgas en conjunto, el grupo de 35-48 tiene $22.2 \%$ de mortalidad fetal, y el grupo de 18 a $34,15.4 \%$; en las cesáreas las cifras, muy altas al parecer, son $16.2 \%$ y $7.7 \%$ respectivamente. Sólo en el fórceps alto y medio, cuya existencia obviamente es de lamentar, pues constituye un procedimiento que ha debido desaparecer, las cifras se invierten. (Cuadro No. 15).

CUADRO No. 15

TIPO DE PARTO Y MORTALIDAD PERINATAL

\begin{tabular}{|c|c|c|c|c|}
\hline \multirow[t]{3}{*}{ TIPO DE PARTO } & \multicolumn{4}{|c|}{ MORTALIDAD FETAL. } \\
\hline & \multicolumn{2}{|c|}{$35-48$} & \multicolumn{2}{|c|}{$18-34$} \\
\hline & $\begin{array}{c}\text { Ante-Intra } \\
\text { Parto }\end{array}$ & Post-Parto & $\begin{array}{c}\text { Ante-Intra } \\
\text { Parto }\end{array}$ & Post-Parto \\
\hline Espontáneo & $4.1 \%$ & $0.9 \%$ & $1.4 \%$ & $0.6 \%$ \\
\hline $\begin{array}{l}\text { Espontáneo con } \\
\text { refuerzo }\end{array}$ & $3.5 \%$ & $0.0 \%$ & $2.0 \%$ & $0.3 \%$ \\
\hline Fórceps & $0.0 \%$ & $0.0 \%$ & $0.9 \%$ & $0.9 \%$ \\
\hline Extracción al vacío & $0.0 \%$ & $10.0 \%$ & $5.3 \%$ & $3.5 \%$ \\
\hline Fórceps alto-medio & $0.0 \%$ & $0.0 \%$ & $3.1 \%$ & $6.3 \%$ \\
\hline Nalgas & $22.2 \%$ & $0.0 \%$ & $9.2 \%$ & $6.2 \%$ \\
\hline Cesárea & $11.7 \%$ & $4.5 \%$ & $6.2 \%$ & $1.5 \%$ \\
\hline Procedimiento & & & & \\
\hline destructivo & $10.0 .0 \%$ & & $100.0 \%$ & \\
\hline
\end{tabular}

FUENTE: G. López, "La E. Añosa", CCRP.PRIF 901

\section{COMENTARIOS}

A la luz de los diversos hallazgos y cuadros, se ha señalado y cuantificado con cifras colombianas, suficientemente representativas y dicientes, el mérito existente para calificar a la embarazada añosa como un riesgo severo en el campo de la atención materno-infantil.

Comparativamente con el grupo más joven, la enfermedad hipertensiva se dobla, el sangrado (particularmente, la hemorragia post-parto)se hace mayor, las cesáreas se duplican, la permanen- cia hospitalaria se alarga. La mortalidad materna fue tres veces mayor y la mortalidad perinatal, aun en el vértice anterior, casi se triplica, siendo desusualmente alta en las cesáreas y en algunos tipos de presentaciones como la de nalgas. Es decir, aún dentro de categorías específicas de modalidades de parto y atención igual o variedades de presentación similares, la mortalidad perinatal se hace ostensiblemente mayor en la embarazada por encima de los 35 años.

Las malformaciones congénitas, particularmente en las mayores de 40 años. 
fueron tres veces más frecuentes. Se ha anotado con razón, que las irisomías 21, 18 y el síndrome de Klinefelter y el de la Triple $x$, se acentúan a medida que aumenta la edad de la mujer, probablemente a causa de la no disyunción de la primera meiosis (2).

Aparte del aparentemente poco uso de la episiotomía, en la presente serie. el único hallazgo que se sale de los patrones y no está de acuerdo con otros autores que han señalado igualmente el mayor riesgo en la mujer embarazada añosa (3), se refiere a la frecuencia de la placenta abruptio. A pesar de que la enfermedad hipertensiva en el grupo presentado fue mucho mayor que en el grupo comparativo más joven, la frecuencia de la abruptio no fue, sin embargo, más alta.

Todas las observaciones anteriores sobre este grupo numeroso de embarazadas por encima de los 35 años, sucedidos en nuestro ambiente colombiano. nos lleva a concluir una y otra vez, cómo en ocasiones anteriores (4) que existe una edad biológica ideal en la mujer para que su función reproductiva se lleve a cabo con el máximo de eficacia y el mínimo de riesgo.

\section{RESUMEN}

Sobre un total de 13.450 casos de parto hospitalario en Colombia, recolectados de marzo a octubre de 1977, el presente trabajo analiza 1.168 de estos casos de embarazadas añosas (se han descartado 10 casos, en la mayoría de las veces por razones de defecto de recolección), de los cuales $884(6.6 \%)$, corresponden a mujeres de 35-39 años y $284(2.1 \%)$ a mujeres de 40 a 48 años. Además, se compara este grupo con 11.040 casos de partos en mujeres de 18 a 34 años, correspondiente al mismo estudio.

Comparativamente con el grupo más joven, la enfermedad hipertensiva se dobla, el sangrado particularmente, la hemorragia post-parto se hace mayor. las cesáreas se duplican, la permanencia hospitalaria se alarga. La mortalidad materna fue tres veces mayor y la mortalidad perinatal aun en el vértice anterior casi se triplica, siendo desusualmente alta en las cesáreas y en algunos tipos de presentaciones como la de nalgas. Es decir, dentro de categorías específicas de modalidades de parto y atención igual o variedades de presentación similares, la mortalidad perinatal se hace obstensiblemente mayor en la embarazada por encima de los 35 años.

A la luz de los diversos hallazgos y cuadros se ha señalado y cuantificado con cifras colombianas, suficientemente representativas y dicientes, el mérito existente para calificar a la embarazada añosa como un riesgo severo en el campo de la atención materno-infan. til.

\section{Summary}

From a totat of 13.450 cases of hospital delivery in Colombia, compiled from March to October, 1977, this paper analysis 1168 of such cases of aged pregnants $(10$ were eliminated due tilack of collection), of which 884 $(6.6 \%)$ correspond to women between 35 and 39 years and $284(2.1 \%)$ to women between 40 and 48 years old. Furthermore, this group is compared to 11.040 delivery cases in women between 18 and 34 years, corresponding to the same study.

In comparison with the younger group, hypertension il!ness is doubled, specially blood letting, post-delivery hemorrhage is higher, caesareans are doubled and hospitalization is longer. Mother mortality was three times higher and perinatal mortality, even in the preceeding vertex, was almost tripled, being unusually high in caesareans and in certain types of presentations such as the rump. In other words, within specific categories of delivery types and equal attention or similar pre- 
sentation varieties, perinatal mortality is clearly higher in a pregnant woman of more than 35 years old.

In the light of the different discoveries and tables, the merit for qualifying the aged pregnant with a high risk in mother and child attention has been quantified and showed in Colombian figures, sufficiently representative and uttering.

\section{BIBLIOGRAFIA}

(1) LOPEZ-ESCOBAR, Guilermo, RIAÑO-GAMBOA, Germán. "El Aborto Hospitalario en Colombia: Estudio de un nuevo grupo de 3.901 casos en hospitales generales no universitarios". Bogotá, D. E., nov. 1979.
(2) K. TSUEJI \& R. Nagano. "Chromosome Studies from Induced Abortions in Pregnant Women aged 35 and over". Obst \& Gynec. V. 52 No. 5 p. 542 nov. 1978.

(3) E. HORGER \& A. R., Smythe. "Pregnancy in Women over Fourty". Obst \& Gynec. V. 49 No. 3: 257, March 1977.

NORTMAN D.. "Parental Age as a Factor in Pregnancy outcome and Child Development" - Reports on Population/Family Planning. Pop. Council No. 16/74.

(4) G. LOPEZ-ESCOBAR. "Salud y Población". Revista Cámara de Comercio de Bogotá. Año 3 No. 12, p. 53, sept/73. 